\title{
Morning home blood pressure may be a significant marker of nephropathy in Japanese patients with type 2 diabetes: ADVANCED-J study 1
}

\author{
Yasushi Tanaka ${ }^{1}$, Hiroyuki Daida ${ }^{2}$, Yutaka Imai ${ }^{3}$, Katsumi Miyauchi ${ }^{2}$, Yasukazu Sato ${ }^{4}$, Masao Hiwatari $^{5}$, \\ Akira Kitagawa $^{6}$, Junji Kishimoto ${ }^{7}$, Tsutomu Yamazaki ${ }^{8}$ and Ryuzo Kawamori ${ }^{2}$
}

A 3-year multicenter, prospective, randomized, open-label trial (ADVANCED-J) compared the effect of an increased dose of angiotensin-II receptor blocker (ARB) with that of a maintenance dose of ARB plus calcium channel blocker (amlodipine) on blood pressure (BP) control, nephropathy and atherosclerosis in patients with type 2 diabetes and hypertension in whom the usual ARB dose failed to control BP. A cross-sectional analysis using baseline data was conducted. Of 316 patients (recruited between September 2004 and December 2005), 228 patients were evaluated by multiple regression analysis using two models after randomization and exclusions. Model 1 assessed 13 baseline variables (age, sex, estimated diabetes duration, estimated hypertension duration, HbA1c, brain natriuretic peptide (BNP), high-sensitive C-reactive protein (hsCRP), triglycerides (TGs), total cholesterol (TCHO), diabetic retinopathy (DMR), systolic morning home BP (HBP), diastolic morning HBP and brachialankle pulse wave velocity (baPWV)) for correlation with the urinary albumin creatinine excretion rate (UACR). In model 2 , systolic and diastolic morning HBP was replaced by systolic and diastolic office BP. The systolic morning HBP and systolic office BP or diastolic morning HBP and diastolic office BP correlations were weak, but significant ( $r=0.43$ and 0.48 , respectively). BNP, $\mathrm{HbA1c}$, DMR and estimated diabetes duration were significantly correlated with UACR in both models 1 and 2. Although systolic office BP did not show a significant correlation with UACR in model 2, systolic morning HBP showed a significant correlation with UACR in model 1 . Morning HBP, but not office BP, may be a significant marker of nephropathy in Japanese patients with type 2 diabetes.

Hypertension Research (2009) 32, 770-774; doi:10.1038/hr.2009.96; published online 26 June 2009

Keywords: morning home blood pressure; nephropathy; office blood pressure

\section{INTRODUCTION}

The combination of hyperglycemia and hypertension markedly increases the risk of diabetic nephropathy in patients with type 2 diabetes. $^{1,2}$ The United Kingdom Prospective Diabetes Study (UKPDS) showed that each $10 \mathrm{~mm} \mathrm{Hg}$ decrease in systolic blood pressure (SBP) was associated with a $12 \%$ reduction in the risk of any diabetes-related complication and with a $13 \%$ decrease in microvascular complications, including nephropathy. ${ }^{3,4}$ Thus, to reduce the risk and/or slow the progression of nephropathy, a strict target blood pressure (BP) $(<130 / 80 \mathrm{~mm} \mathrm{Hg})$ has been recommended. ${ }^{5}$ As 24 -h ambulatory BP monitoring (ABPM) can now be easily performed with reliable monitoring devices, a number of studies have shown the inadequacy of evaluating BP control from clinical measurements alone. ${ }^{6,7}$ Recent studies have indicated that home BP (HBP) measured in the morning has a stronger predictive power for mortality than does office BP measurement. ${ }^{8,9}$ These reports suggest the importance of monitoring morning HBP, in addition to office BP, to evaluate BP control and to adjust antihypertensive therapy. However, earlier large-scale prospective studies of diabetic complications, such as the UKPDS, ${ }^{2-4}$ only monitored office BP, and the association between morning HBP and nephropathy was not fully evaluated.

\footnotetext{
${ }^{1}$ Department of Medicine, St Marianna University School of Medicine, Kawasaki, Japan; ${ }^{2}$ Department of Medicine, Juntendo University School of Medicine, Tokyo, Japan; ${ }^{3}$ Department of Clinical Pharmacology and Therapeutics, Tohoku University Graduate School of Pharmaceutical Sciences and Medicine, Sendai, Japan; ${ }^{4}$ Department of Internal Medicine, Takagi Hospital, Medical Corporation of Kouhoukai, Ohkawa, Japan; ${ }^{5}$ Division of Physical Therapy, Graduate School, University of Health and Welfare, Ohtawara, Japan; ${ }^{6}$ Department of Clinical Trial Management, International University of Health and Welfare Graduate School, Tokyo, Japan; ${ }^{7}$ Digital Medicine Initiative, University of Kyushu, Fukuoka, Japan and ${ }^{8}$ Department of Clinical Epidemiology and Systems, University of Tokyo Graduate School of Medicine, Tokyo, Japan

This work was supported by the Japan Heart Foundation.

Correspondence: Dr Y Tanaka, Department of Medicine, Metabolism and Endocrinology, St Marianna University School of Medicine, 2-16-1 Sugao, Miyamae-ku, Kawasaki, Kanagawa 216-8511, Japan.

E-mail: y2tanaka@marianna-u.ac.jp

Received 28 April 2009; revised 28 May 2009; accepted 28 May 2009; published online 26 June 2009
} 
The ADVANCED-J study is a 3-year prospective trial designed to compare the effect of increasing the dose of an angiotensin-II receptor blocker (ARB) on BP control, nephropathy and atherosclerosis with that of adding the dihydropiridine calcium channel blocker (DCCB; amlodipine) in hypertensive Japanese patients with type 2 diabetes in whom BP control using the usual ARB dose was inadequate. ${ }^{10}$ Both office $\mathrm{BP}$ and morning $\mathrm{HBP}$ were monitored. A unique point of this study was setting the BP target on the basis of morning HBP, not office BP. Antihypertensive therapy was adjusted to obtain a morning HBP $<125 / 80 \mathrm{~mm} \mathrm{Hg}$ during the study. In this analysis, to determine the relationship between morning HBP and nephropathy, a cross-sectional assessment of the correlation between the urinary albumin/creatinine ratio (UACR) and morning $\mathrm{HBP}$ or office $\mathrm{BP}$ was conducted using baseline data from the ADVANCED-J study.

\section{METHODS}

\section{Study design and participants}

This study was a cross-sectional analysis of the data obtained from the ADVANCED-J study, a multicenter, prospective, randomized, open-label trial conducted over 3 years in 300 patients with type 2 diabetes and ARB-resistant hypertension. The study protocol was approved by the ethics committee of each study site. All patients gave written informed consent to participate. Briefly, 316 patients were recruited during the registration period from 22 centers, whereas office BP was monitored for at least 8 weeks with treatment at the usual single daily dose of an ARB (candesartan $8 \mathrm{mg}$, losartan $50 \mathrm{mg}$, telmisartan $40 \mathrm{mg}$, valsartan $80 \mathrm{mg}$ or olmesartan $20 \mathrm{mg}$ ). The final part of the registration period was a control period with ARB alone, lasting for at least 2 weeks, during which time measurement of morning HBP was carried out by patients with a systolic office BP $\geqslant 135 \mathrm{~mm} \mathrm{Hg}$ or a diastolic office BP $\geqslant 85 \mathrm{~mm} \mathrm{Hg}$. Treatment was started when the mean morning HBP for the preceding 5 days of the control period on ARB alone was $\geqslant 130 \mathrm{~mm} \mathrm{Hg}(\mathrm{SBP})$ or $\geqslant 80 \mathrm{~mm} \mathrm{Hg}$ diastolic blood pressure (DBP). A total of 263 eligible patients were allocated to two study groups on the basis of the minimization method, controlling for their urinary albumin level during the control period on ARB alone $(<300 v s . \geqslant 300 \mathrm{mg}$ per gram creatinine) and for systolic HBP (SHBP) $(<135 \mathrm{~mm} \mathrm{Hg}$ vs. $\geqslant 135 \mathrm{~mm} \mathrm{Hg}$ ) in the morning after waking, but before starting the study. They either received a higher dose of the same ARB up to the maximum approved dose (candesartan $12 \mathrm{mg}$, losartan $100 \mathrm{mg}$, telmisartan $80 \mathrm{mg}$, valsartan $160 \mathrm{mg}$ or olmesartan $40 \mathrm{mg}$; ARB group, $n=132$ ), or continued to receive $\mathrm{ARB}$ at the earlier dose plus amlodipine at $5 \mathrm{mg}$ daily (combination group, $n=131$ ). Primary clinical end points were changes in home BP after arising in the morning and the achievement rate of a home BP level of $<125 \mathrm{~mm} \mathrm{Hg}$ systolic and $<80 \mathrm{~mm} \mathrm{Hg}$ diastolic. The determination of sample size was based on the assumption that treatment differences in change from baseline BP were estimated to be at least $5 \pm 15 \mathrm{~mm} \mathrm{Hg}$ (mean \pm s.d.) for SBP and DBP. It was calculated that, to achieve an $80 \%$ or higher power on the primary end point, 142 patients per arm (a total of 284 patients) would be needed to show the superiority of the ARB group using a two-sided test at an $\alpha$-level of 0.05 .

\section{Measurement of variables and analysis}

During the control period on ARB alone of at least 2 weeks, morning HBP and evening HBP were measured by patients. HBP data obtained with an electronic home digital BP monitor were transmitted to the central computer server in Tokyo through a portable telemetry device connected to the monitor, and data were automatically input into the software program for analyses (CapTool, a semi-automated electronic data capture system; Mebix, Tokyo, Japan). Patients were instructed to measure their HBP after resting for $5 \mathrm{~min}$ in the sitting position, after waking in the morning and before going to bed. Compliance with measurement could be monitored using the software. To exclude the influence of incorrect measurement, SBP > $200 \mathrm{~mm} \mathrm{Hg}$ and DBP $<40 \mathrm{~mm} \mathrm{Hg}$ were defined as errors and rejected by this system. Office BP was also measured at the outpatient clinic at least three times by a physician during this period, that is, at the beginning of the control period on ARB alone, during the study period and at its end. Measurements were obtained after $5 \mathrm{~min}$ of rest in the sitting position. Laboratory tests (fasting blood sample) included testing for HbAlc, plasma glucose, insulin, brain natriuretic peptide (BNP), highsensitive C-reactive protein (hsCRP), triglycerides (TGs), total cholesterol (TCHO), blood urea nitrogen (BUN) and creatinine. In addition, the urinary albumin creatinine excretion rate (UACR), chest X-ray, ECG, carotid ultrasound, B-mode echocardiography and brachial-ankle pulse wave velocity (baPWV) were examined at least once during the control period on ARB alone of 2 weeks or longer.

\section{Table 1 Clinical characteristics of patients}

\begin{tabular}{|c|c|c|}
\hline & \multicolumn{2}{|c|}{$\mathrm{n}=263(M 162 / F 101)$} \\
\hline & Mean \pm s.d. & (n) \\
\hline Age (years) & $65.1 \pm 9.7$ & 263 \\
\hline $\mathrm{BMI}\left(\mathrm{kg} \mathrm{m}^{-2}\right)$ & $25.3 \pm 3.8$ & 263 \\
\hline Estimated duration of hypertension (years) & $6.8 \pm 7.6$ & 247 \\
\hline Estimated duration of diabetes (years) & $7.1 \pm 6.8$ & 253 \\
\hline Systolic clinic BP (mm Hg) & $151.4 \pm 15.5$ & 263 \\
\hline Diastolic clinic BP $(\mathrm{mm} \mathrm{Hg})$ & $83.1 \pm 11.2$ & 263 \\
\hline Systolic morning BP (mm Hg) & $157.8 \pm 17.9$ & 263 \\
\hline Diastolic morning BP (mm Hg) & $83.5 \pm 11.7$ & 263 \\
\hline HbAlc (\%) & $6.79 \pm 0.97$ & 259 \\
\hline $\mathrm{BNP}\left(\mathrm{pg} \mathrm{ml^{-1 }}\right)$ & $46.3 \pm 58.1$ & 239 \\
\hline hsCRP (mg per $100 \mathrm{ml}$ ) & $0.17 \pm 0.24$ & 240 \\
\hline TG (mg per $100 \mathrm{ml}$ ) & $145.7 \pm 96.4$ & 258 \\
\hline TCHO (mg per $100 \mathrm{ml}$ ) & $199.1 \pm 33.4$ & 258 \\
\hline Creatinine (mg per $100 \mathrm{ml}$ ) & $0.81 \pm 0.25$ & 259 \\
\hline UACR (mg per g Cr) & $263.7 \pm 676.9$ & 263 \\
\hline $\mathrm{EF}(\%)$ & $68.9 \pm 8.1$ & 248 \\
\hline$E / A$ ratio & $0.88 \pm 0.29$ & 226 \\
\hline IVS-d (mm) & $11.0 \pm 2.3$ & 248 \\
\hline Right mean IMT (mm) & $0.84 \pm 0.20$ & 202 \\
\hline Left mean IMT (mm) & $0.88 \pm 0.24$ & 201 \\
\hline PW-d (mm) & $10.9 \pm 1.9$ & 248 \\
\hline CTR (\%) & $49.6 \pm 4.7$ & 243 \\
\hline Right baPWV $\left(\mathrm{cm} \mathrm{s}^{-1}\right)$ & $1820 \pm 377$ & 231 \\
\hline Left baPWV $\left(\mathrm{cm} \mathrm{s}^{-1}\right)$ & $1833 \pm 362$ & 230 \\
\hline Right ABI & $1.13 \pm 0.13$ & 231 \\
\hline Left $A B I$ & $1.11 \pm 0.13$ & 232 \\
\hline
\end{tabular}

Abbreviations: baPWV, brachial-ankle pulse wave velocity; BMI, body mass index; BP, blood pressure: BNP, brain natriuretic peptide; $\mathrm{Cr}$, creatinine; $\mathrm{EF}$, ejection fraction; $\mathrm{F}$, female; hsCRP, pressure; BNP, brain natriuretic peptide; Cr, creatinine; $\mathrm{EF}$, ejection fraction; F, female; hsCRP,
high-sensitive C-reactive protein; M, male; TG, triglyceride; TCHO, total cholesterol; UACR, high-sensitive $\mathrm{C}$-reactive protein; $\mathrm{M}$, male;
urinary albumin creatinine excretion rate.

Table 2 Characteristics of patients $\mathrm{n}=263(M 162 / F 101)$

(n) $\%$

\begin{tabular}{lrr}
\hline No. of patients with a history of & & \\
Cerebrovascular disease & 24 & 9.1 \\
Coronary heart disease & 40 & 15.2 \\
Cardiac failure & 3 & 1.1 \\
& & \\
No. of patients using anti-diabetic drugs & & \\
Sulfonylureas & 96 & 36.5 \\
Glinides & 14 & 5.3 \\
Biguanides & 37 & 14.1 \\
Pioglitazone & 26 & 9.9 \\
$\alpha$-Glucosidase inhibitors & 66 & 25.1 \\
Insulin & 54 & 20.5
\end{tabular}

Abbreviations: F, female; M, male. 


\section{Data analysis}

Correlation between morning HBP and office BP was evaluated by a linear regression analysis. To assess the influence of morning HBP on nephropathy, the correlation between UACR and morning HBP or office BP was evaluated by multiple regression analysis using two models. Model 1 examined the association between UACR (objective variable: logarithmic transformation) and 13 variables, baseline hsCRP, BNP, HbAlc, TG, TCHO, diabetic retinopathy (DMR), SBP and DBP for morning HBP (morning SHBP and morning DHBP), baPWV, sex, age, estimated duration of hypertension and estimated duration of diabetes (explanatory variables). In model 2, a correlation with UACR (logarithmic transformation) was assessed using SBP and DBP for office BP (office SBP and office DBP) instead of morning SHBP and morning DHBP. These two models were used to analyze 228 patients, as data for 35 patients were incomplete. All analyses were carried out using SAS statistical software (version 9.1; SAS Institute Inc., Cary, NC, USA), and a $P$-value of $<0.05$ was considered to be statistically significant.
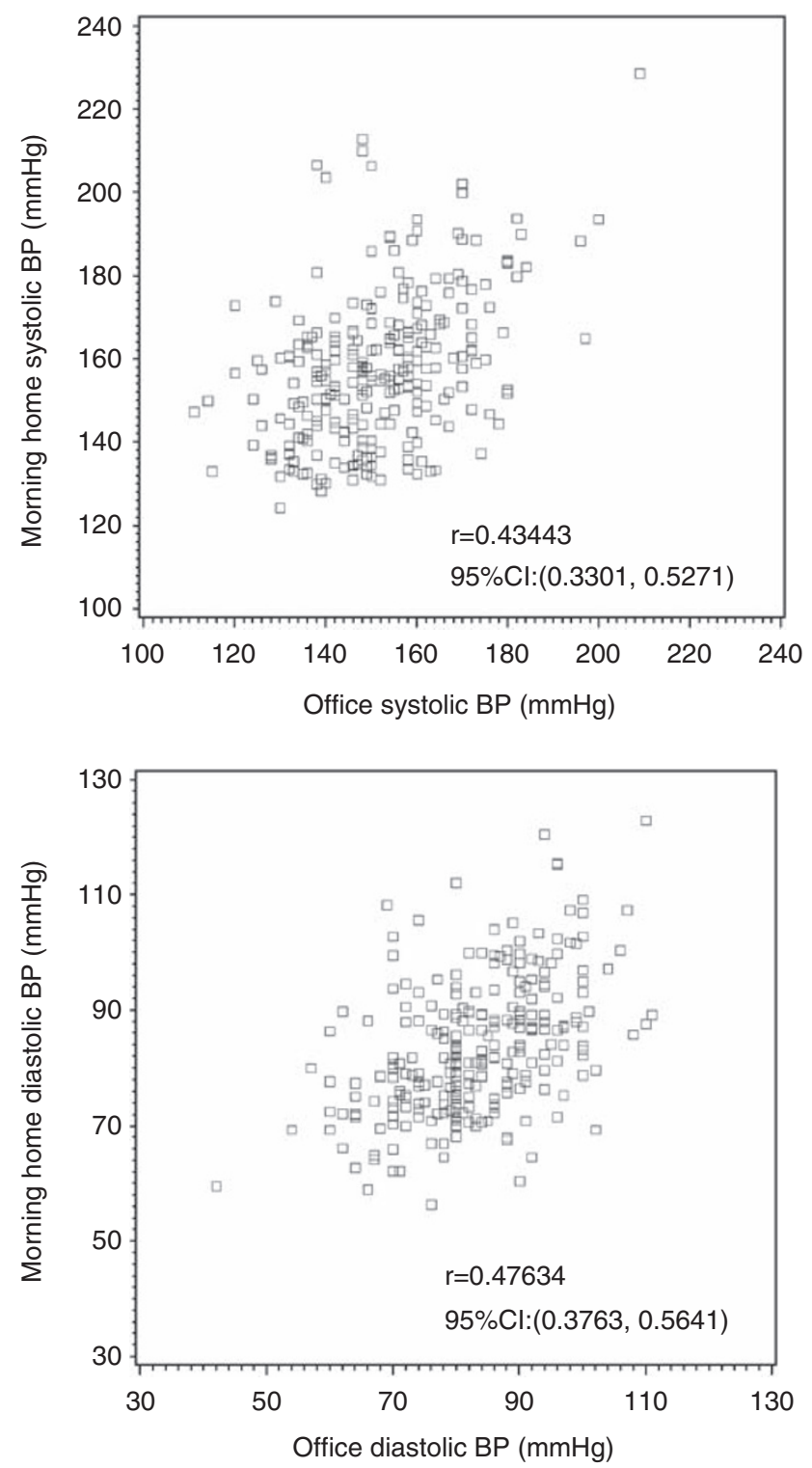

Figure 1 Correlation between morning BP and office BP. Abbreviations: $\mathrm{BP}$, blood pressure; UACR, urinary albumin creatinine excretion ratio; $\mathrm{Cl}$, confidence interval; $r$, pearson's product-moment correlation coefficient.

\section{RESULTS}

The characteristics of eligible patients were randomized as shown in Tables 1 and 2. The 263 patients comprised 162 men and 101 women with a mean age of 65 years (range, 35-85 years). As shown in Figure 1, the correlation between morning SHBP and office SBP or between morning DHBP and office DBP was significant, although weak, with coefficients ( $r$ values) of 0.43 and 0.48 , respectively. Table 3 shows the association between UACR (logarithmic transformation) and other variables in the two models, evaluated by multiple regression analysis. In model 1, BNP, HbAlc, TG, TCHO, DMR, morning SHBP, estimated duration of diabetes and sex were initially selected by stepwise analysis. As a result of re-analysis using these eight variables,

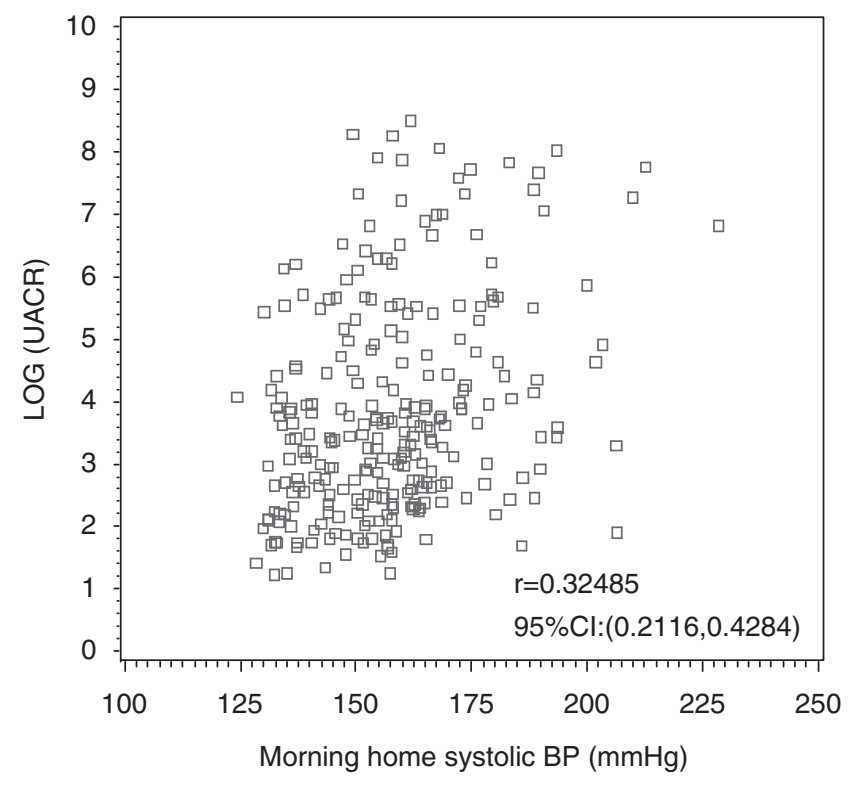

UACR (logarithmic transformation) and Morning home systolic BP plot

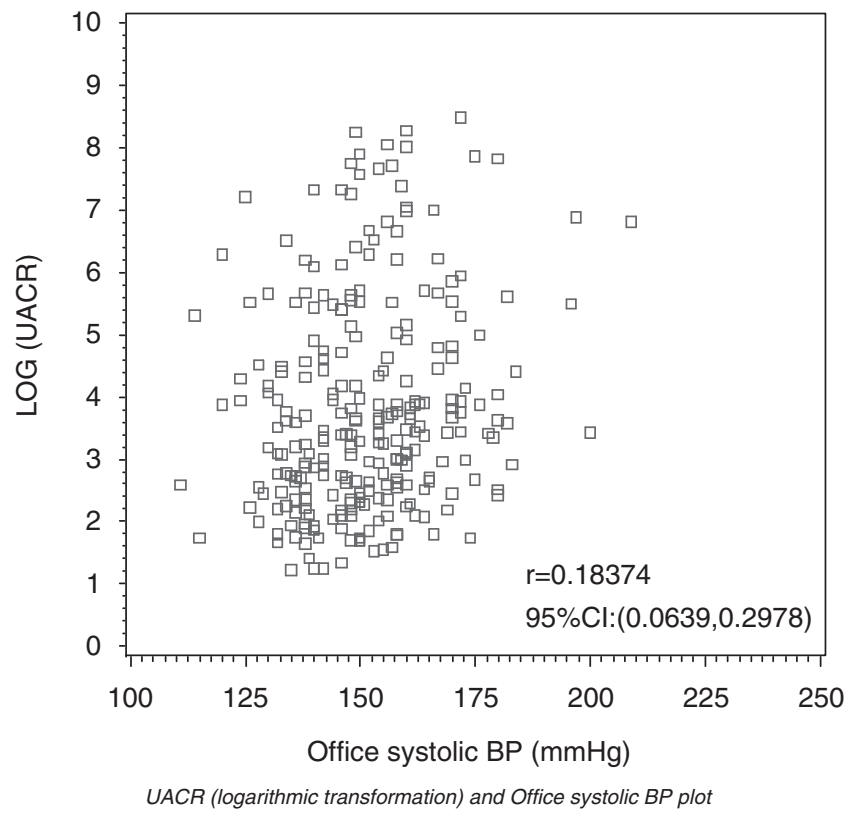

Figure 2 Correlation between $\log [\mathrm{UACR}]$ and systolic BP. Abbreviations: $\mathrm{BP}$, blood pressure; UACR, urinary albumin creatinine excretion ratio; $\mathrm{Cl}$, confidence interval; $r$, pearson's product-moment correlation coefficient. 
Table 3 Correlation of clinical factors with UACR on multiple regression analysis

\begin{tabular}{|c|c|c|c|c|}
\hline Variable & Partial regression coefficient & $\begin{array}{c}\text { Standard partial regression } \\
\text { coefficient }\end{array}$ & P-value & $95 \% \mathrm{Cl}$ \\
\hline \multicolumn{5}{|l|}{ Model-1 } \\
\hline $\mathrm{HbAlc}(\%)$ & 0.347 & 0.198 & 0.001 & $0.139-0.555$ \\
\hline TG (mg per $100 \mathrm{ml}$ ) & 0.001 & 0.079 & 0.195 & $-0.001-0.004$ \\
\hline TCHO (mg per $100 \mathrm{ml}$ ) & 0.006 & 0.120 & 0.054 & $0.000-0.012$ \\
\hline Estimated duration of diabetes (years) & 0.036 & 0.134 & 0.023 & $0.005-0.067$ \\
\hline Sex & -0.410 & -0.113 & 0.053 & $-0.827-0.006$ \\
\hline \multicolumn{5}{|l|}{ Model-2 } \\
\hline $\mathrm{BNP}\left(\mathrm{pg} \mathrm{ml}^{-1}\right)$ & 0.005 & 0.166 & 0.007 & $0.001-0.008$ \\
\hline $\mathrm{HbA1c}(\%)$ & 0.324 & 0.185 & 0.004 & $0.104-0.544$ \\
\hline baPWV & 0.001 & 0.146 & 0.022 & $0.000-0.001$ \\
\hline Estimated duration of diabetes (years) & 0.051 & 0.191 & 0.003 & $0.018-0.084$ \\
\hline Sex & -0.433 & -0.119 & 0.056 & $-0.877-0.011$ \\
\hline
\end{tabular}

Abbreviations: baPWV, brachial-ankle pulse wave velocity; BNP, brain natriuretic peptide; Cl, confidence interval; DMR, diabetic retinopathy; TG, triglyceride; TCHO, total cholesterol.

Model 1 examined the association between UACR (objective variable:logarithmic transformation) and 13 variables, baseline hsCRP, BNP, HbA1c, TG, TCHO, DMR, morning SHBP, morning DHBP, baPWV, sex, age, estimated duration of hypertension, and estimated duration of diabetes (explanatory variables). In model 2, correlation with UACR (Iogarithmic transformation) was assessed using office SBP and office DBP instead of morning SHBP and morning DHBP.

BNP, HbAlc, DMR, morning SHBP, estimated duration of diabetes, and sex showed a significant relationship with UACR. In model 2, BNP, HbAlc, TG, TCHO, DMR, office SBP, baPWV, estimated duration of diabetes and sex were initially selected, and BNP, HbAlc, DMR, baPWV and estimated duration of diabetes showed a significant association with UACR (logarithmic transformation) on re-analysis. In particular, a strong relation $(P<0.0001)$ was observed between UACR (logarithmic transformation) and morning SHBP in model 1. The correlation coefficient between UACR (logarithmic transformation) and morning SHBP was $r=0.32$, and the correlation coefficient between UACR (logarithmic transformation) and office SHBP was $r=0.18$ in Figure 2. Log transformation was used to reduce right skewness in the distribution for UACR before statistical analyses in Table 3 and Figure 2.

\section{DISCUSSION}

This study showed weak, but significant, correlations between morning SHBP and office SBP or between morning DBP and office DBP, whereas BNP, HbA1c, DMR and morning SHBP (but not office SBP) were significantly correlated with UACR in diabetic patients who had hypertension resistant to the standard ARB therapy.

Earlier studies have shown an elevation of BNP level in type 2 diabetic patients with microalbuminuria or macroalbuminuria compared with that in patients with normoalbuminuria, and results from this study are consistent with these findings. ${ }^{11,12}$ However, those studies and current study data included patients with coronary heart disease (CHD). Recently, Igarashi et al. ${ }^{13}$ reported similar results for type 2 diabetic patients, but they observed no difference between patients with normoalbuminuria and those with microalbuminuria/ macroalbuminuria after excluding patients with CHD. Thus, in this study, multiple regression analysis was performed after excluding patients with $\mathrm{CHD}$ and cerebrovascular disease. HbA1c, DMR and morning SHBP still showed a significant correlation with UACR, but not BNP. This result suggests that BNP may be associated with progression of macrovascular disease, such as CHD or cerebrovascular disease, but not with albuminuria.

To date, most large-scale studies addressing the effect of antihypertensive therapy on morbidity and mortality have been based on office $\mathrm{BP}, 2,14,15$ and the role of HBP monitoring in managing hypertension remains unclear. Against this background, results from this study suggest the importance of home-based BP monitoring for detection and/or control of nephropathy, and that reliance on clinic-based BP measurement alone may hamper the timely identification of patients with incipient nephropathy. These findings are consistent with those of an earlier study of the significance of hypertension assessed by morning HBP measurement for the development of nephropathy and atherosclerosis in diabetic patients. Kamoi et al. ${ }^{16}$ measured clinic BP once during each visit and once each morning at home for 1 month in 170 type 2 diabetic patients receiving antidiabetic and antihypertensive therapy. They defined clinic and home morning hypertension $(\mathrm{MH})$ as $\mathrm{SBP} \geqslant 130 \mathrm{~mm} \mathrm{Hg}$ and/or DBP $\geqslant 85 \mathrm{~mm} \mathrm{Hg}$. Although there were no significant differences in the prevalence of nephropathy, retinopathy, CHD and cerebrovascular disease between groups with $(n=131)$ and without clinic hypertension $(n=39)$, the prevalence of these complications was significantly higher $(P<0.05)$ in patients with $(n=97)$ than in those without $(n=73) \mathrm{MH}$. In particular, nephropathy was strongly associated with systolic $\mathrm{MH}$.

Interestingly, Nielsen et al..$^{17}$ reported that the prevalence of whitecoat hypertension (clinical hypertension with a normal BP at home on 24-h ABPM) was significantly higher in diabetic patients with normoalbuminuria (urinary albumin excretion (UAE) $<30 \mathrm{mg}$ per day) than in those with microalbuminuria (UAE; $30-300 \mathrm{mg}$ per day) or macroalbuminuria (UAE $>300 \mathrm{mg}$ per day), that is, $23 \mathrm{vs}$. $8 \%$ and $9 \%$, respectively. These reports suggest that office BP may not exactly 
reflect the state of 24-h BP in diabetic patients, and that it may be difficult to detect patients with incipient nephropathy by monitoring office BP alone. Recently, Leitão et al. ${ }^{18}$ reported that type 2 diabetic patients with masked hypertension (normal office BP and elevated HBP detected by 24-h ABPM) have a higher UAE than those without masked hypertension. Taken together, these findings suggest that office BP may not be related to HBP, and HBP monitoring may be more useful for evaluating the risk of nephropathy than is office BP. However, these studies included many patients without antihypertensive therapy, whereas all patients in this study were resistant to the standard ARB therapy. Thus, it is difficult to compare these studies directly with the current study, and it remains unclear why the correlation between morning HBP and office BP was weak or why morning HBP, but not office BP, was correlated with UACR in this study. Further investigation will be required to clarify these issues.

There are several weaknesses in this study, including the fact that it was a cross-sectional study, and that an HBP monitor, not a 24-h ABPM system, was used. Also, UACR was only measured once during the control period on ARB alone. As the ADVANCED-J study involves 265 patients and is a unique prospective trial focusing on morning $\mathrm{BP}$ adjusted with antihypertensive therapy to obtain a target morning HBP $<125 / 80 \mathrm{~mm} \mathrm{Hg}$, an ordinary HBP monitor, rather than an ABPM system, was used. The data transfer system and CapTool allowed measured precise BP data to be exactly transmitted to the central server, and major system problems have not occurred to date. The ADVANCED-J study is still in progress, therefore the relationship between UAE and morning HBP, plus the significance of normalizing morning BP to prevent the development and/or progression of nephropathy, may be clarified further by future data.

In conclusion, a significant association of UCAR with morning SHBP in diabetic patients with hypertension refractory to conventional ARB therapy was identified, whereas no association was found with office BP. Thus, morning BP may be a significant marker of nephropathy in Japanese patients with type 2 diabetes.

1 American Diabetes Association. Nephropathy in diabetes. Diabetes Care 2004; 27 (Suppl 1): S79-S83.

2 Stratton IM, Cull CA, Adler AI, Matthews DR, Neil HAW, Holman RR. Additive effects of glycaemia and blood pressure exposure on risk of complications in type 2 diabetes: a prospective observational study (UKPDS 75). Diabetologia 2006; 49: 1761-1769.

3 Adler AI, Stratton IM, Neil HA, Yudkin JS, Matthews DR, Cull CA, Wright AD, Turner RC, Holman RR. Association of systolic blood pressure with macrovascular and microvas- cular complications of type 2 diabetes (UKPDS 36): prospective observational study. Br Med J 2000; 321: 412-419.

4 UK Prospective Diabetes Study Group. Tight blood pressure control and risk of macrovascular and microvascular complications in type 2 diabetes. $\mathrm{Br}$ Med J 1998; 317: 703-713.

5 American Diabetes Association. Hypertension management in adults with diabetes. Diabetes Care 2004; 27(Suppl 1): S65-S67.

6 Aihara A, Imai Y, Sekino M, Kato J, Ito S, Ohkubo T, Tsuji I, Satoh H, Hisamichi S, Nagai K. Discrepancy between screening blood pressure and ambulatory blood pressure: a community-based study in Ohasama. Hypertens Res 1998; 21: 127-136.

7 Vollmer WM, Appel LJ, Svetkey LP, Moore TJ, Vogt TM, Conlin PR, Proschan M, Harsha D. DASH Collaborative Research Group, comparing office-based and ambulatory blood pressure monitoring in clinical trials. J Hum Hypertens 2005; 19: 77-82.

8 Ohkubo T, Imai Y, Tsuji I, Nagai K, Kato J, Kikuchi N, Nishiyama A, Aihara A, Sekino M, Kikuya $M$, Ito $S$, Satoh $H$, Hisamichi $S$. Home blood pressure measurement has a stronger predictive power for mortality than does screening blood pressure measurement: a population-based observation in Ohasama, Japan. J Hypertens 1998; 16: 971-975.

9 Gorostidi M, Sobrino J, Segura J, Sierra C, de la Sierra A, Hernández del Rey R, Vinyoles E, Galcerán JM, López-Eady MD, Marín R, Banegas JR, Sarría A, Coca A, Ruilope LM. Spanish Society of Hypertension ABPM Registry Investigators, ambulatory blood pressure monitoring in hypertensive patients with high cardiovascular risk: a cross-sectional analysis of a 20,000-patient database in Spain. J Hypertens 2007; 25: 977-983.

10 Kawamori R, Daida H, Tanaka Y, Miyauchi K, Kitagawa A, Hayashi D, Kishimoto J, Ikeda S, Imai Y, Yamazaki T. Amlodipine versus angiotensin II receptor blocker; control of blood pressure evaluation trial in diabetics (ADVANCED-J). BMC Cardiovasc Disord 2006; 6: 39.

11 Yano Y, Katsuki A, Gabazza EC, Ito K, Fujii M, Furuta M, Tuchihashi K, Goto H, Nakatani K, Hori Y, Sumida Y, Adachi Y. Plasma brain natriuretic peptide levels in normotensive noninsulin-dependent diabetic patients with microalbuminuria. J Clin Endocrinol Metab 1999; 84: 2353-2356.

12 Nagai T, Imamura M, Inukai T, Mori M. Brain natriuretic polypeptide in type 2 NIDDM patients with albuminuria. J Med 2001; 32: 169-180.

13 Igarashi M, Jimbu Y, Hirata A, Tominaga M. Characterization of plasma brain natriuretic peptide level in patients with type 2 diabetes. Endocr J 2005; 52: 353-362.

14 ALLHAT Officers and Coordinators for the ALLHAT Collaborative Research Group. Major outcomes in high-risk hypertensive patients randomized to angiotensin-converting enzyme inhibitor or calcium channel blocker vs. diuretics: the Antihypertensive and Lipid-Lowering Treatment to Prevent Heart Attack Trial (ALLHAT). JAMA 2002; 288: 2981-2997.

15 Dahlöf B, Devereux RB, Kjeldsen SE, Julius S, Beevers G, de Faire U, Fyhrquist F, Ibsen $\mathrm{H}$, Kristiansson K, Lederballe-Pedersen $\mathrm{O}$, Lindholm LH, Nieminen MS, Omvik P, Oparil S, Wedel H. LIFE study group, cardiovascular morbidity and mortality in the Losartan intervention for endpoint reduction in hypertension study (LIFE): a randomized trial against atenolol. Lancet 2002; 359: 995-1003.

16 Kamoi K, Miyakoshi M, Soda S, Kaneko S, Nakagawa O. Usefulness of home blood pressure measurement in the morning in type 2 diabetic patients. Diabetes Care 2002; 25: 2218-2223.

17 Nielsen FS, Gaede P, Vedel P, Pedersen O, Parving HH. White coat hypertension in NIDDM patients with and without incipient and overt diabetic nephropathy. Diabetes Care 1997; 20: 859-863.

18 Leitão CB, Canani LH, Kramer CK, Boza JC, Pinotti AF, Gross JL. Masked hypertension, urinary albumin excretion rate, and echocardiographic parameters in putatively normotensive type 2 diabetic patients. Diabetes Care 2007; 30: 1255-1260. 